\title{
Research on Data Processing of Robot Tactile Sensing Costume
}

\author{
Bing Guo ${ }^{1, a^{*}}$, Xinli Deng ${ }^{1, b}$ \\ Chongqing Radio \& TV University, Chongqing 400052, China \\ a*cqguobing@126.com, bouodeng@tom.com
}

Keywords: surgical robot tactile, cognitive map, object recognition.

\begin{abstract}
Aiming at the difficulties to make data processing by description in graph of structure and characteristic, and at the limitations resulting in interference and feedback mechanism for data processing of robot tactile sensing garment, the paper presented a novel fuzzy cognitive map based data processing model. In this paper, we defined the basic structure model of fuzzy cognitive map, studied on modelling process of cognitive map, discussed the constructing steps of tactile cognitive maps, derived and presented the algorithm of fuzzy cognitive map based on tactile shape recognition for data processing. Taking a robot composed of 32 pieces of $4 \times 16$ tactile sensor array as an example, through the real time acquisition of tactile signals by LabVIEW, the simulation experiment of Matlab fuzzy cognitive map demonstrated that it could simultaneously and intuitively display the distribution of the stress, the distribution profile of different pressure range and the distribution and size of the pressure peak for each sensing point. The results of simulation experiment prove that the presented fuzzy cognitive map based data processing model is feasible, and it is more clear and concise than conventional data processing method.
\end{abstract}

\section{Introduction}

Principle robot haptic tactile sensor is in contact with the object to be identified or interaction to complete the surface characteristics and physical properties of perception [1] . Robot tactile sensing costume refers to the distribution of clothing in the robot body type tactile sensor, tailoring and sewing can be processed in accordance with the shape of the robot, the robot can be worn on the body perception of environmental information. Its main feature is the large surface of the flexible tactile sensor shape and unrestricted feeling multifunctional.

Robot tactile sensing costume has the following characteristics: (1) obtain the location, pressure and other data through the sensor. Sensing data on the performance of two-dimensional spatial information. Clothing robot tactile sensing is passive touch, similar to machine vision. May seek to use the image processing method.

Take time pressure data, the spatial distribution. (2) Compared with machine vision, tactile information is more diversity. In addition to the spatial data and pressure distribution data, the robot tactile sensor can be obtained by contacting the substance 
Variety of physical information, such as the surface roughness of the contact material, temperature, hardness, material, etc. [2] . This is similar to human skin function, you can achieve a richer environment for robot perception, and is conducive to human-computer interaction information.

Robot touch are the 1960s emerging haptic research, in so far proposed intelligent robot tactile sensing method, some use polyvinylidene fluoride (PVDF) film piezoelectric effect to obtain pressure information, some using ultrasound [3] or pressure caused by the change in capacitance, as well as the use of mechanical switches, optical waveguides [4] and other programs, but these programs are mostly considered tactile sensing technology used in robot hands, fingers [5], there are individual proposals for the robot arm joints. Currently distributed in the robot body type clothing tactile sensing technology [6] less study than at home and abroad. Herein developed intelligent robot tactile sensing costume, not only has high sensitivity and large area, etc., can also detect contact with objects in contact with the robot body contour and surface pressure distribution, the results could be used for the body humanoid robot tactile sensing system to achieve new breakthroughs in technology robot sensing.

\section{DATA ACQUISITION AND DATA PROCESSING}

Tactile sensing costume for each sensor block access to lead the ranks of multi-channel analog switch, through multi-channel analog switch block selection. Selected blocks the sensor voltage signal of each sensor unit sequentially fed signal conditioning circuits, blocks selected host control signal will have a good conditioning according to the progressive scan mode to capture the host, you will be sensitive to the various units tactile sensor information data block. Signal acquisition robot tactile sensing costume system block diagram.

Processing data comprising: sensing costume receive stimuli from the environment, to obtain haptic data; sensor data for filtering, edge detection preprocessing; touch object shape recognition processing; tactile sensations processing.

If the 8-bit A / D tactile signals as the A / D conversion device, the haptic $16 \times 16$ array of $16 \times 16$ pixels, 256 gray levels tactile image. Each pixel corresponds to a tactile dot gradation represents the tactile pressure on values. Thus, like the tactile image processing visual signal processing, you can get some information about the contact with the object by its handle. Filtering many ways, the most common is the median filtering, tactile image filtered with good analytical performance. Signal processing, the following parameters can be obtained from the tactile image: pressure, contact area, the tactile image center position, azimuth angle. It can also be obtained from the slip by multi-frame dynamic changes tactile images.

Clothing haptic data processing tasks can be divided into two categories, one touch object recognition and display appearance, one intended tactile understanding. Contact with objects shape processing can be applied to the moment of inertia image feature recognition method normalized after acquiring a contact material appearance, tactile intention to build cognitive maps can be processed and the corresponding reasoning completed. Cognitive map structured knowledge ease of description, containing explicit knowledge of causality is more suitable expression [7] . Cognitive map by combining adjacent matrix and the weight coefficient of the knowledge representation, reasoning through the matrix operation to achieve. Intent processing can be done by constructing cognitive maps 
and the corresponding reasoning. Cognitive map structured knowledge ease of description, containing explicit knowledge of causality is more suitable expression [7] . Cognitive map by combining adjacent matrix and the weight coefficient of the knowledge representation, reasoning through the matrix operation to achieve. Fig. 1 shows the structure of robot tactile processing.

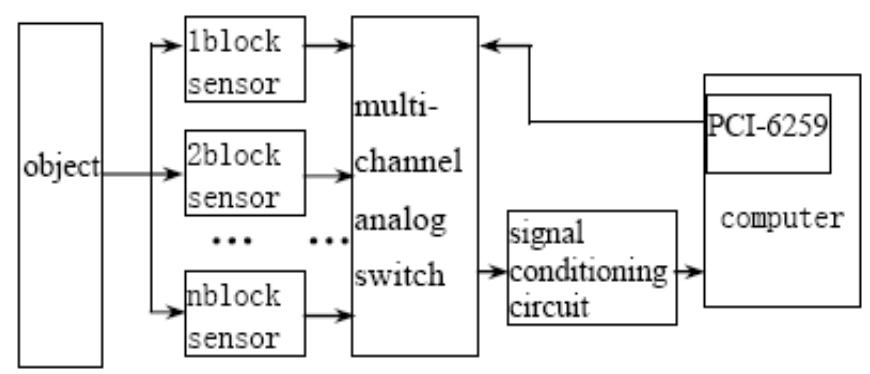

Fig. 1 Structure of robot tactile processing

Fig. 2 shows the outline of tactile data processing. As the identifying process, conversion and compensation process are practiced with data processing unit. At the beginning of this process, raw tactile data are converted with sensor conversion unit to the robot's surface. Next the robot compensates the displacement of the coordinate systems of the robot's body. The data processing unit is consisted of three processes. The first process is sensor conversion unit which represents the correspondence of the tactile data to the robot's body. The second one is position compensation unit which stores rules of the dynamic change of sensor conversion process. The third is self-interference compensation unit. The self-interference data is related to the robot's posture, therefore this unit is relatiive between the self-interference data and the angle of each robot's link and is utilized to compensate the self-interference. Using self-interference compensation unit and the robot's posture, the robot detects self-interference areas in the neighborhood of the bending joints such as elbows, knees. To acquire self-interference compensation data, the robot detects the change of tactile data while moving a certain bending joint such as the elbows, and stores the relationship between the area of the change in sensor and the angle of that joint. The processed sensor data is stored as symbolized tactile data, which is utilized to generate the robot behavior. The symbolized tactile data has data structure which is made by mapping the distributed tactile data onto the robot's surface [5] .

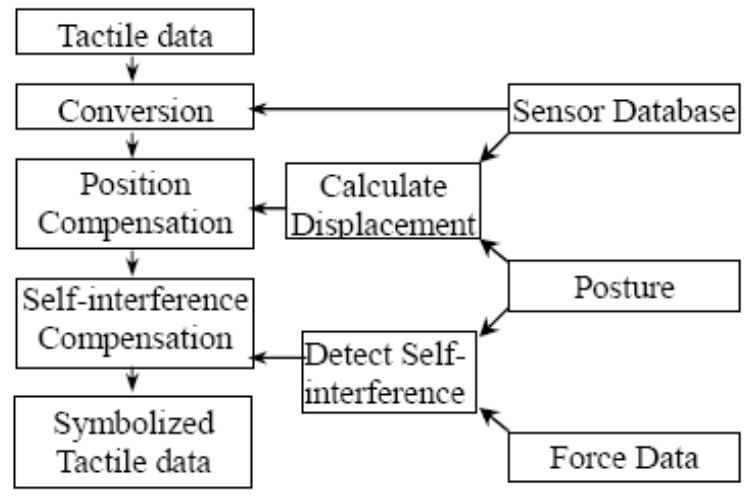

Fig. 2 Conversion and compensation of tactile data

The task of tactile data process duty may divide into two kinds. One is the recognition and display of the shape, the other is the understanding of tactile intention. The method of normalized moment of inertia can be used for shape recognition. Tactile intention process can be accomplished by 
constructing cognitive map. Structure knowledge is easy to describe by cognitive map. Cognitive map is more suitable for knowledge expression that contains the obvious causal relation. The knowledge expression and the inference process are realized through the operation on the adjacency matrix.

\section{THE CONCEPT OF COGNITIVE MAP}

A cognitive map is a representation of relationships which are perceived to existence among the elements of a given environment [6] . It is a concept causal relation graph in knowledge expression and inference system. The concept is expressed as node and the causal relation is expressed as the edge.

[Definition 1] concept and its status value

Concept is depicted as a node. It also is called concept node. Concept node can express system operation, cause, and result and trend and so on. $\mathrm{Ci}$ denotes concept node. Concept status values denotes Vci. Causal relation is represented as an edge.

[Definition 2] Causality value

Cognitive map edge value between two concepts, $\mathrm{i}$ and $\mathrm{j}$, written by Wij, indicates the causality value between the two concepts. Causality values lies between $[-1,1]$.

$\mathrm{Wij}=0$ means no causal relation between concepts $\mathrm{i}$ and $\mathrm{j}$;

Wij > 0 indicates causal increase (positive causality);

Wij $<0$ indicates causal decrease (negative causality);

[Definition 3] Weight matrix

An Adjacency matrix E, based on the causality value $\{\mathrm{Wij}, \ldots . . ., \mathrm{Wik} . . . .$.$\} between the$ concepts .

All steps of building a tactile cognitive map include:

1) Confirm concept node set of the direct graph according the object characteristic $\mathrm{C}=\{\mathrm{C} 1, \mathrm{C} 2, \ldots \ldots, \mathrm{Ck}\}$

2) Confirm the cause relation is existed in the concept node set $\mathrm{C}=\{\mathrm{C} 1, \mathrm{C} 2, \ldots \ldots, \mathrm{Ck}\}$. Connect the concept nodes which have cause relation with direct edge; 
3) Establishment related weight matrix with expert judgment methods;

4) Threshold operation

$C_{i}(t+1)=\left\{\begin{array}{l}1, \text { if } \mathrm{C}_{\mathrm{i}}(\mathrm{t}) \mathrm{E}_{\mathrm{i}}>0 \\ 0, \text { if } \mathrm{C}_{\mathrm{i}}(\mathrm{t}) \mathrm{E}_{\mathrm{i}} \leq 0\end{array}\right.$

The key step is the selection of concept node and the setup of weight matrix.

\section{SHAPE RECOGNITION}

Format of tactile sensor data The data from sensor may include coordinate value L (xi,yi) and the press data Pi or material data. Data set $\mathrm{S}(\mathrm{L}, \mathrm{P} \ldots \ldots$...) is composed of two-dimension digital images $(M \times N)$.

The method of shape recognition Two-dimension digital images $(M \times N)$ may be viewed as a $X O Y$ two-dimensional surface pixel. Each pixel gradation value (pressure value etc.) is $f(x, y)$. Images sequence (xi, yi) are recognized using methods of normalized moment of inertia recognition [7] .

$$
\begin{aligned}
& f(x, y) \text { Mass center }\left(C_{x}, C_{y}\right) \\
& C_{x}=\frac{\sum_{x=1}^{M} \sum_{y=1}^{N} x \times f(x, y)}{\sum_{x=1}^{M} \sum_{y=1}^{N} f(x, y)} \\
& C_{y}=\frac{\sum_{x=1}^{M} \sum_{y=1}^{N} y \times f(x, y)}{\sum_{x=1}^{M} \sum_{y=1}^{N} f(x, y)}
\end{aligned}
$$

Moment of inertia around Mass center $\left(C_{x}, C_{y}\right)$ denotes $J_{\left(C_{x}, C_{y}\right)}$ :

$$
\begin{aligned}
J_{\left(C_{x}, C_{y}\right)} & =\sum_{x=1}^{M} \sum_{y=1}^{N}\left[(x, y)-\left(C_{x}, C_{y}\right)\right]^{2} f(x, y) \\
& =\sum_{x=1}^{M} \sum_{y=1}^{N}\left(\left(x-C_{x}\right)^{2}+\left(y-C_{y}\right)^{2}\right) f(x, y)
\end{aligned}
$$


Around $\left(C_{x}, C_{y}\right) \mathrm{NMI}($ Normalized Moment of Inertia):

$$
\begin{aligned}
& N M I=\frac{\sqrt{J_{\left(C_{x}, C_{y}\right)}}}{m}=\frac{\sqrt{\sum_{x=1}^{M} \sum_{y=1}^{N}\left(\left(x-C_{x}\right)^{2}+\left(y-C_{y}\right)^{2}\right) f(x, y)}}{\sum_{x=1}^{M} \sum_{y=1}^{N} f(x, y)} \\
& m=\sum_{x=1}^{M} \sum_{y=1}^{N} f(x, y) \quad \text { is image mass. }
\end{aligned}
$$

NMI has good shrinking and enlarging, and rotary and translation immutability. NMI algorithm is simple, the operand is small, good RST immutability. NMI is one of important method in robot tactile data processing.

Basics shape database is pre-installed in the data process system. Characteristic information of basics shape such as rectangle, round, finger is stored in the database. The NMI distance between object and basics shape is used for measuring the similarity by methods of k-Nearest Neighbor Classifiers.

\section{TACTILE PERCEPTION}

Tactile cognitive map If one pats another on the back with the hand, it expresses the friendliness. If he lashes him, it is attack. The robot perception through tactile sensor is similarly so. It's judge the intention come from another according to the shape, the action position, the force, and the action time etc and makes the response [8] [9] .

Robot perception through tactile sensor is similarly so. Concept node expresses lots of meaning such as shape, action position, force, action time, kindness, threat, attack, angry, politeness, happiness, etc. Shape is the result of tactile image procession. There are direct causal relation between shape (or action position or force or action time) and kindness (or threat or attack), between kindness and happiness [10] [11] . Causality value and threshold are established according to the expert experience.

The tactile cognitive map is shown in Fig. 3 and will be explained in the following. 


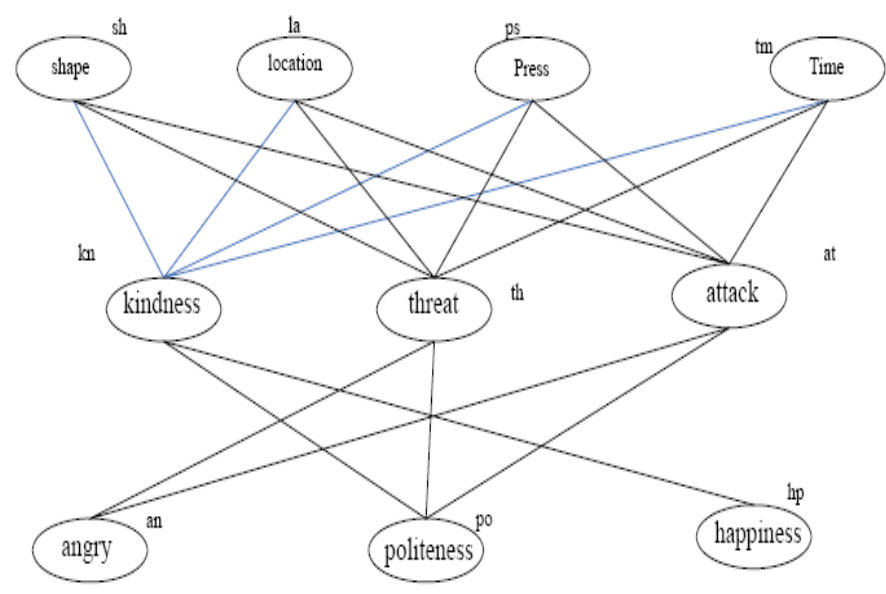

Fig. 3 Cognitive map of robot tactile

Concept node and weight matrix (initial value) In general, an cognitive map with $n$ concept nodes is described by an $n \times n$ adjacency matrix, $E$, whose elements are the causal link strengths (or weights) $e i j$. The matrix corresponding to the example cognitive map with ten concepts shown in Fig.4 is:

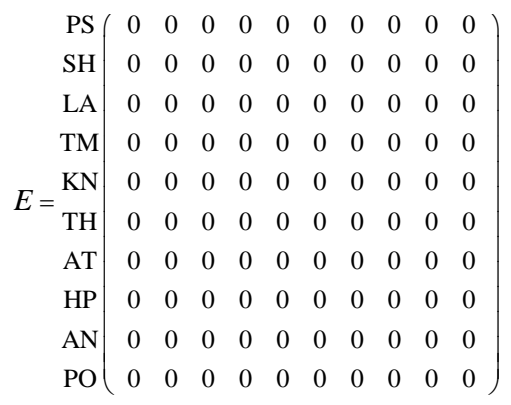

Fig. 4 Adjacency matrix

The element in the ith row and $j$ th column of matrix $\boldsymbol{E}$ represents the strength of the causal link from $C i$ to $C j$. The concept values of nodes $C 1, C 2, \ldots, C n$ (where $n$ is the number of concepts in the problem domain) together represent the state vector $C$.

\section{Algorithm description}

Step 1: Get the row vector that represents the concept;

Step 2: Decide on a threshold value in the interval $[-1,1]$;

Step 3: Multiply the row vector by adjacency matrix $\boldsymbol{E}$;

Step 4: Compare the values received to the threshold.

\section{EXPERMENTS WITH ROBOT TACTILE}


Connection of the system debugging, robot costume four tactile sensor array composed of $32 \times$ 16. Tactile sensing clothing and pre-scan circuitry, signal conditioning circuitry, and NI data acquisition card companies PCI6259 connection with LabVIEW real-time acquisition complete tactile signals, and a voltage signal acquisition card to obtain spatial coordinates sensing array of data, and the data is stored at the specified address, the data read by the VC program and use PFCM haptic tactile shape cognitive map method is determined by the display program to reconstruct the shape of the contact contour of lattice points in the color depth means that the size of the external pressure.


Fig.5 Multi-point pressed and the result
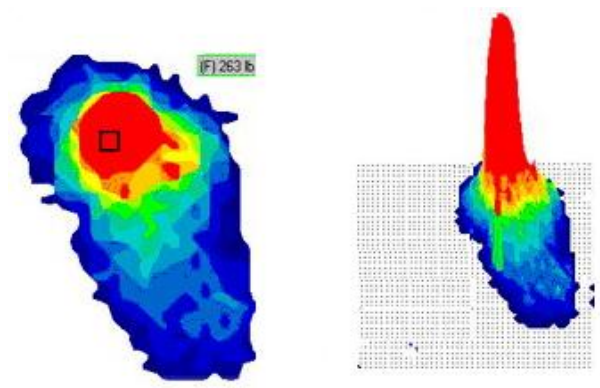

Fig.6 2-D and 3-D views of peak pressure

Fig.5 and Fig.6 show the experimental result. From the experimental results can be seen, the robot tactile sensing costume experimental system can accurately reflect the tactile information. When the contact is contacted for multiple ranks (multi-point) on the sensor array can be obtained according to the data acquisition and processing of the outline structure of the contact material in accordance with the red dot and the color depth to determine the size of each pressure point, while the two-dimensional and three-dimensional display display and visualize the forces in different pressure ranges and pressure distribution profile of peaks and size distribution of each robot tactile sensing costume point, clear and concise. Therefore verify that the design is feasible.

\section{Conclusions}

The robot perception to environment through tactile sensor suits is passive. Another type tactile sense is through the initiative touching to gain environment information, such as robot finger touching object. The object recognition is based on 3-D recognition. Similarly we can make up cognitive map of initiative tactile. Perceive information and reaction can be gained by operation. While cognitive map is the common computation platform, it is advantageous to fuse the above two kinds of information. Unifying with the machine vision, the robot perceive may be more perfective.

\section{Acknowledgment}


This research supported by Program for Excellent Talents in Chongqing Higher Education Institutions.

\section{References}

[1] LUO Zhizeng,WANG Rencheng, "Study of tactile sensor in bionical artificial hand", Chinese Journal of Sensors and Actuayors,2003,16(3):233-237.

[2] M. H. Lee, H. R. Nicholls, "Tactile sensing for mechatronics-A state of the art survey", Mechatron, 1999, vol. 9:1-31.

[3] Shigeru Ando, Hiroyuki Shinoda, "Ultrasonic emission tactile sensor for contact localization and characterization", IEEE International Conference on Robotics and Automation, 1994: 2536-2543.

[4] Yukiko HOSHINO, Masayuki INABA,and Hirochika INOUE, "Model and Processing of Whole-body Tactile Sensor Suit for Human-Robot Contact Interaction ”, IEEE International Conference on Robotics and Automation, 1998:2281-2286.

[5] Carvalho, J.P., Tomé, J.A., "Qualitative Optimization of Fuzzy CausalRule Bases using Fuzzy Boolean Nets", Fuzzy Sets and Systems,158(17), pp. 1931-1946, Elsevier, 2007

[6] Carvalho, J.P., Tomé,J.A., "Views on Fuzzy Sets and Systems fromDifferent Perspectives. Philosophy and Logic, Criticisms andApplications. (Studies in Fuzziness and Soft Computing), Chapter 19 -Fuzzy Mechanisms for Qualitative Causal Relations”, Edited by.Rudolf Seising, Springer, 2009

[7] Carvalho, J.P., Tomé, J.A., "Rule Based Fuzzy Cognitive Maps -Qualitative Systems Dynamics", Proceedings of the 19th InternationalConference of the North American Fuzzy Information ProcessingSociety, NAFIPS2000, Atlanta, 2000

[8] Carvalho, J.P., Tomé, J.A.,"Issues on the Stability of Fuzzy CognitiveMaps and Rule-Based Fuzzy Cognitive Maps", Proceedings of the 21stInternational Conference of the North American Fuzzy InformationProcessing Society, NAFIPS2002, New Orleans, 2002

[9] M. Ohka, Y. Mitsuya, and S. Takeuchi,et al, "A three-axis optical tactile sensor", IEEE International Conference on Robotics and Automation, 1995:817-824.

[10] PAN Zengxi, CUI Hongliang, and ZHU Zhenqi, "A flexible full-body tactile sensor of low cost and minimal connections", IEEE International Conference on Volume 3, Issue, 2003: 2368-2373.

[11] LUMELSKY V J, SHUR M S, and WAGNER S, "Sensitive skin", IEEE Sensors Journal, 2001:1(1): 41-51.

[12] Luo Xiangfeng, "Cognitive map theory and its applications in image analysis and understanding", Hefei: Hefei university of technology, 2003:12-20.

[13] W. Zhang, S. Chen, "A Logical architecture for cognitive maps", Proceedings of the 2nd IEEE conference on neural networks, vol.I, 1988:231-238.

[14] YANG Xiaogang, FU Guangyuan and MIAO Dong, "A new approach to target recognition based on image NMI feature", Computer Engineering, 2002, 24(6): 79-82.

[15] LIUT Zhi-oiang, MIAO Yuan, "Fuzzy cognitive map and its causal inferences", IEEE International Fuzzy Systems Conference Proceedings, Seoul, Korea: IEEE 1999:22-25. 